\title{
Phenylalanine and tyrosine transfer RNAs encoded by Tetrahymena pyriformis mitochondrial DNA: primary sequence, post-transcriptional modifications, and gene localization
}

\author{
Murray N. Schnare ${ }^{1}$, Taisto Y. K. Heinonen ${ }^{2}$, Paul G. Young ${ }^{2}$, and Michael W. Gray ${ }^{1}$ \\ 1 Dept. of Biochemistry, Dalhousie University, Halifax, Nova Scotia B3H 4H7, Canada \\ 2 Department of Biology, Queen's University, Kingston, Ontario K7L 3N6, Canada
}

Current Genetics (1985) 9:389-393

Due to an unfortunate error, the country in the first affiliation was incorrect as originally printed. It should have read as above. 